\title{
Review: Modeling damping in mechanical engineering structures
}

\author{
Michel Lalanne \\ INSA, Laboratoire de Mécanique des Structures, \\ UPRESA CNRS 5006, 20 av Albert Einstein, 69621 \\ Villeurbanne, France \\ Tel.: +33 472438231; Fax: +33 472438930; E-mail: \\ michel.lalanne@lmst.insa-lyon.fr
}

Received 22 March 1999

Revised 28 July 1999

This paper is concerned with the introduction of damping effects in the analysis of mechanical engineering structures. Damping can be considered as being generated by concentrated elements, by distributed elements, or by several effects existing simultaneously. Modeling damping for different engineering situations is described and some applications are presented briefly.

\section{Introduction}

During the design of engineering structures, numerical models are used to ensure satisfactory dynamic behavior. Natural frequencies, modes, frequency and time responses, are dependent on damping which can be quite difficult to model. This paper presents mechanical engineering structures studied at Laboratoire de Mécanique des Structures (LMSt) whose damping was due to concentrated elements, to distributed elements or to several simultaneous effects, and discusses analysis methods employed to take into account damping.

\section{General procedure for modeling structural dynamics}

Equations of the dynamic behavior of mechanical engineering structures are generally obtained by using Lagrange's equations associated with a numerical method used in most computer softwares, the finite element method. Consequently it is necessary to know the kinetic and strain energies and the virtual work of the external forces. Thus, in the best and most frequent case, modeling leads to a set of differential linear equations which can be written as

$$
M \ddot{x}+C(\Omega) \dot{x}+K x=F(t)
$$

where $M$ and $K$ are the mass and stiffness matrices, $C(\Omega)$ contains the gyroscopic matrix and a viscous damping matrix, $x$ and $F(t)$ are the nodal displacement and force vectors.

When dealing with basic vibrations, damping is presumed to be viscous. The reason for this assumption is that the differential set of equations of the system can be easily solved, and an equivalent viscous damping adequately represents damping effects in certain cases. However a major problem concerning the prediction of the dynamic behavior of engineering structures is that damping cannot be often represented in this fashion. In general the system (1) can be considered as linear for a large range of operating conditions. The first step is to solve the free motion equations which give the natural frequencies, associated mode shapes and damping ratio. A second step is concerned with the response of the system to excitation forces. In most cases it is necessary to reduce the number of degrees of freedom of the structure either by a substructure, or by a modal method, and two kinds of calculations are performed on the reduced systems: a frequency response and a time response [12].

\section{Examples of modeling damping due to concentrated elements}

Rotating machinery includes rotors which are commonly supported by hydrodynamic bearings. Their characteristics are obtained from the solution of Reynolds equations [3] which gives stiffness and viscous damping coefficients. Many practical results are given in [15], where for several kinds of bearings, the reduced coefficients, $k_{i j}$ and $c_{i j}$, are given as a function of the Sommerfeld number: 


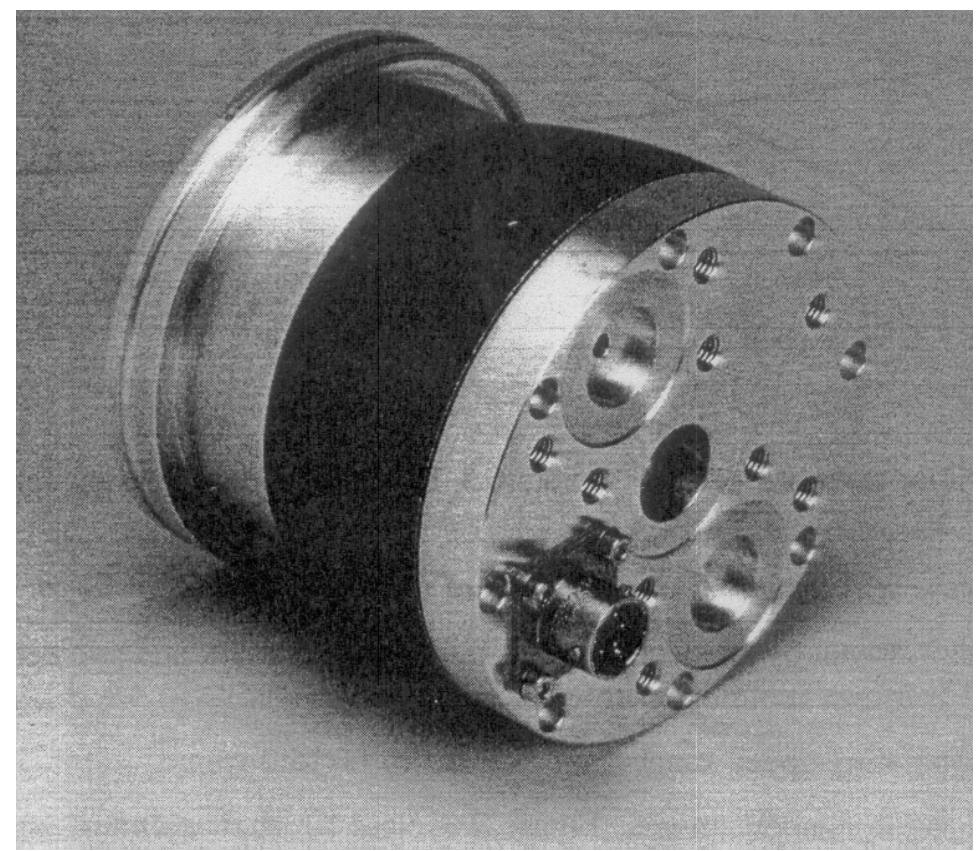

Fig. 1. Turbomolecular pump - Courtesy ALCATEL-CIT.

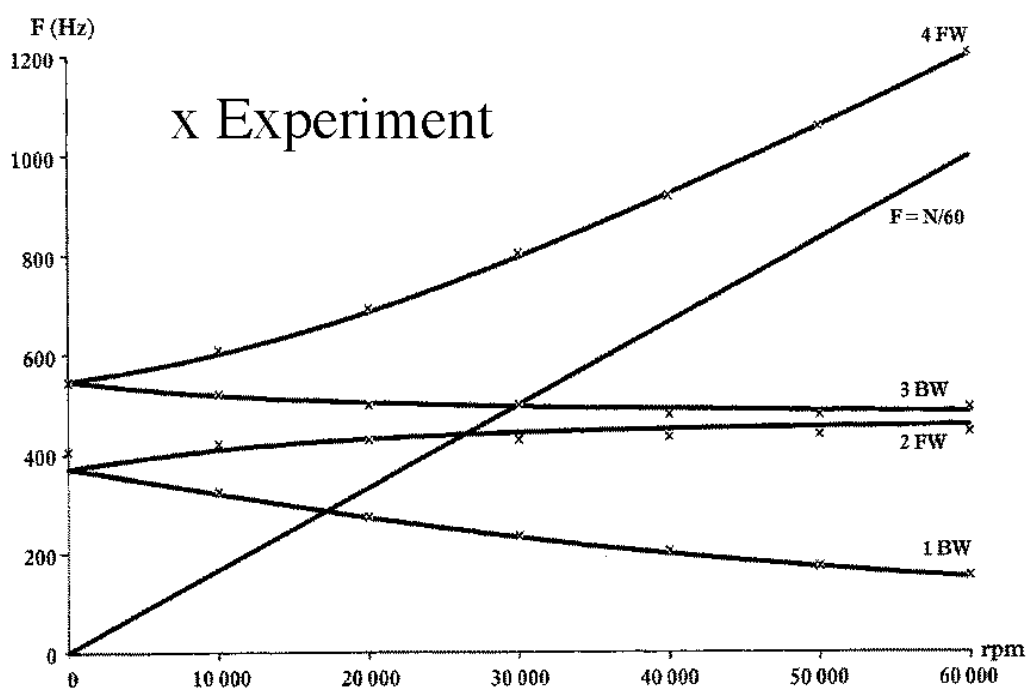

Fig. 2. Campbell diagram.

$$
S=\frac{\mu N L D}{W}\left(\frac{R}{C}\right)
$$

where $\mu$ is the viscosity of the lubricant, $N(\Omega=2 \pi N)$ is the speed of rotation, $R(D=2 R)$ and $L$ are the radius and the length of the bearing, $C$ is the radial clearance and $W$ is the bearing radial load. Thus the stiffness and damping coefficients are:

$$
\begin{aligned}
& K_{i j}=k_{i j} \frac{W}{C} \\
& C_{i j}=c_{i j} \frac{W}{C \Omega}
\end{aligned}
$$

and can be directly included in equations $[6,13]$.

Reference [14] presents the analysis of the turbomolecular pump shown in Fig. 1. The rotor is mounted on two bearings consisting of a roller bearing supported 


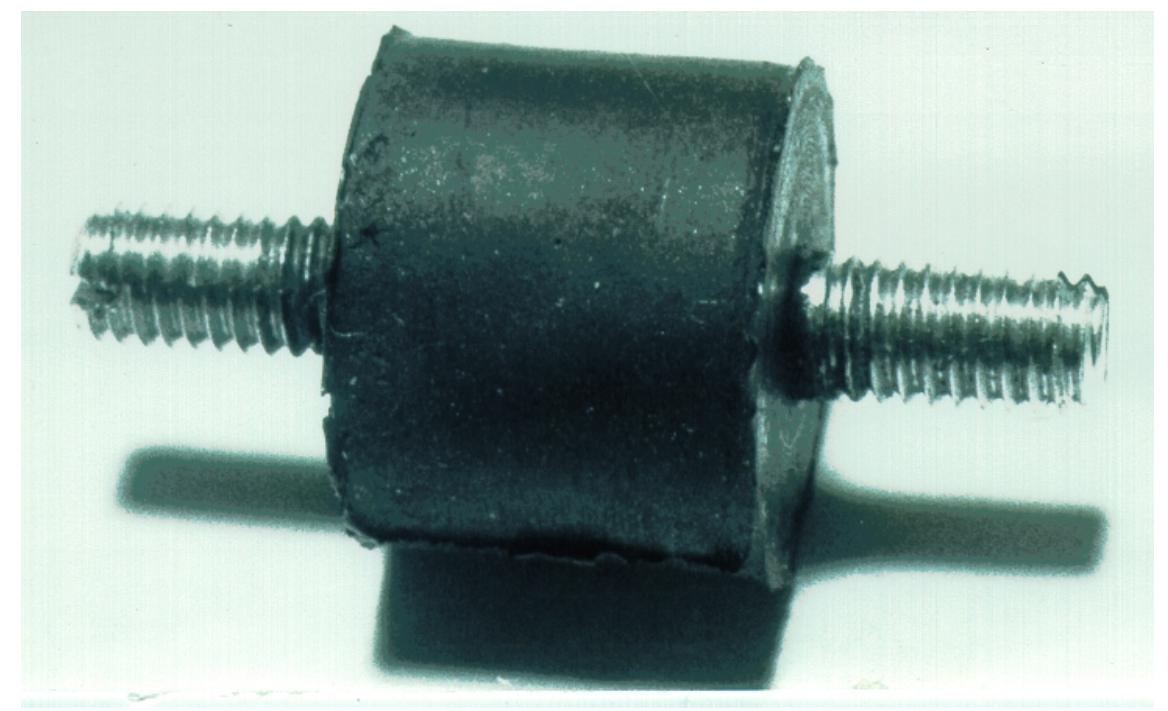

Fig. 3. Elastomer mount.

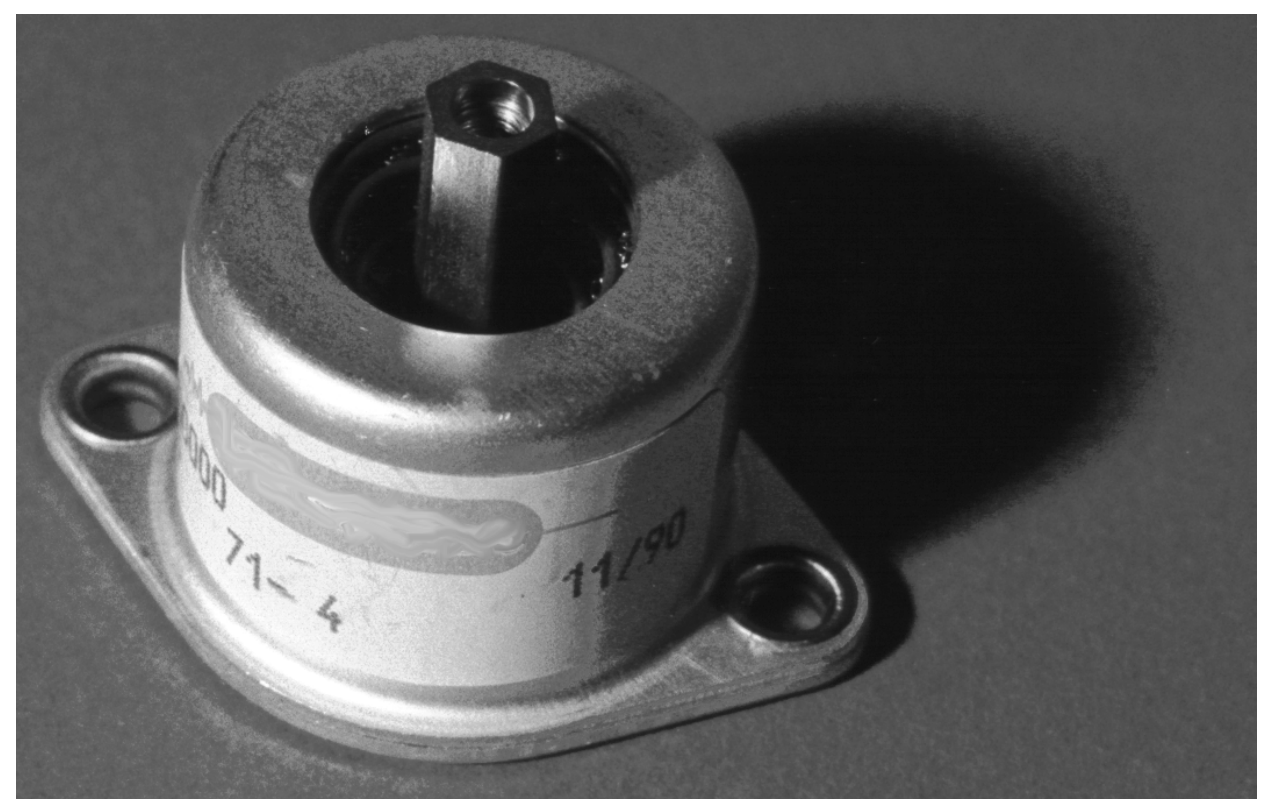

Fig. 4. All-metal mount.

on an elastomer o-ring. The operating speed of rotation ranges from 40000 to $60000 \mathrm{rpm}$. The Campbell diagram which gives the critical speeds was calculated using estimates on the bearing and o-ring assembly stiffness. Then the turbopump rotating up to $60000 \mathrm{rpm}$ was subjected to a harmonic excitation force fixed in space. This frequency response at certain speeds of rotation $(10000-20000, \ldots, 60000 \mathrm{rpm})$ gives all the frequencies shown on the Campbell diagram, i-e the backward $(\mathrm{BW})$ and forward $(\mathrm{FW})$ frequencies. The values of stiffnesses and modal dampings at $20000 \mathrm{rpm}$ were used for calculating the Campbell diagram. Figure 2 shows that experimental and finite element results are in very good agreement.

The elastomer mount and the all-metal mount, shown in Figs 3 and 4 are commonly used for the vibration isolation of structures. Their non linear behavior which arises from material and geometrical design depends on many parameters: temperature, pre-load, forcing frequency and especially deflection for the latter. Their 


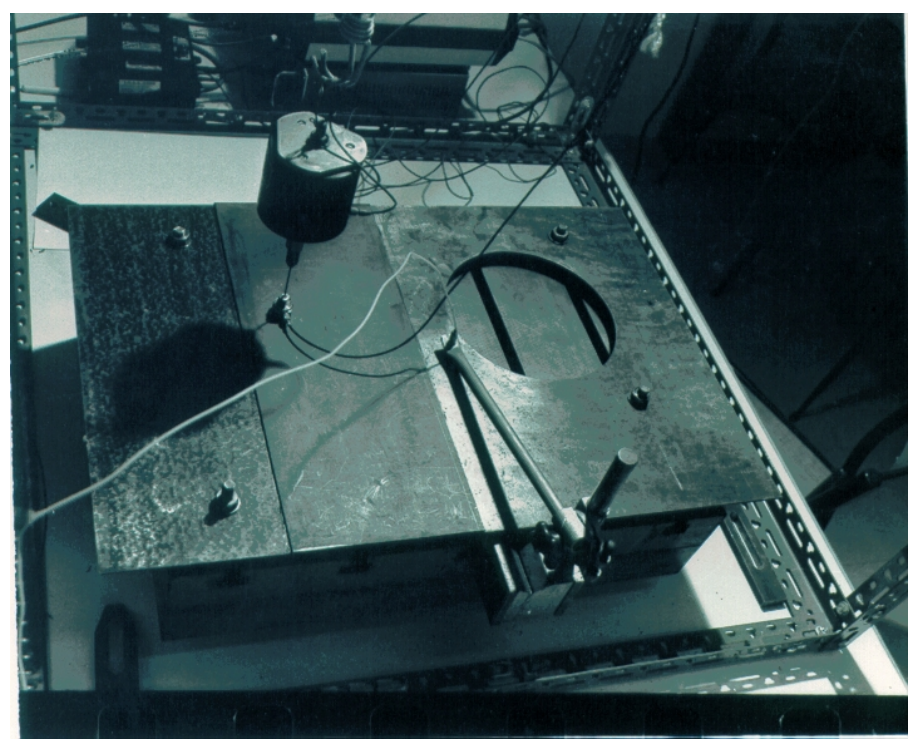

Fig. 5. Test structure.

effect can be modeled either with stiffness or restoring force models by the use of experimental characterization.

For harmonic response the stiffness model is based on the effective stiffness and loss factor which are measured from a harmonic load deflection loop [9]. For time response the stiffness model requires tangent and instantaneous stiffnesses measured from the curve fitting of a quasi-static load deflection loop, the damping being taken into account by the modal damping [8]. Consequently, the stiffness models depend on the type of excitation and cannot be used in the case of superposition of different types of excitation. In such a situation the restoring force model is necessary even if it requires a difficult and tedious experimental characterization. The equations of the structure are coupled with an additional first order differential equation sometimes involving a lot of parameters in order to obtain the hysteretic load-deflection loop as accurately as possible [1].

\section{Examples of modeling damping due to distributed elements}

The applications presented here deal with elements constituted by viscoelastic materials which are frequently a part of a structure. The presentation which follows concerns a non-rotating structure with one viscoelastic material and subjected to a harmonic excitation.
Complex representations of the applied force and displacements are:

$$
\begin{aligned}
& F(t)=F_{o} e^{j \Omega t} \\
& x=X_{o} e^{j \Omega t}
\end{aligned}
$$

thus the frequency response of the system is given by the solution of

$$
\left(-\Omega^{2} M+j \eta_{v} K_{v}+K\right) X_{o}=F_{o}
$$

where $K_{v}$ is the viscoelastic material stiffness matrix and $\eta_{v}$ the loss factor. The number of degrees of freedom of the system is greatly reduced by using the $n$ lowest modes, $\phi_{1}, \ldots, \phi_{n}$, of the associated undamped structure. Hence a new set of coordinates results from:

$$
X_{0}=\phi_{1} q_{1}+\ldots+\phi_{n} q_{n}=\phi_{q}
$$

and the use of equations (7) and (8) gives:

$$
\begin{aligned}
& \left(-\Omega^{2} \phi^{t} M \phi+j \eta_{v} \phi^{t} K_{v} \phi+\phi^{t} K \phi\right) q \\
= & \phi^{t} F_{o}
\end{aligned}
$$

Equation (9) is solved for given values of $\Omega$ and the displacements of the structure result from Equation (8). The advantage is also in the values of the global loss factor, as in many cases the modes can be considered as uncoupled, which can be obtained by:

$$
\eta_{g i}=\eta_{v i} \frac{\phi_{i}^{t} K_{v} \phi_{i}}{\phi_{i}^{t} K \phi_{i}}
$$

where $\eta_{g i}$ and $\eta_{v i}$ are the global and material loss factors corresponding to mode $i[10,11]$. 


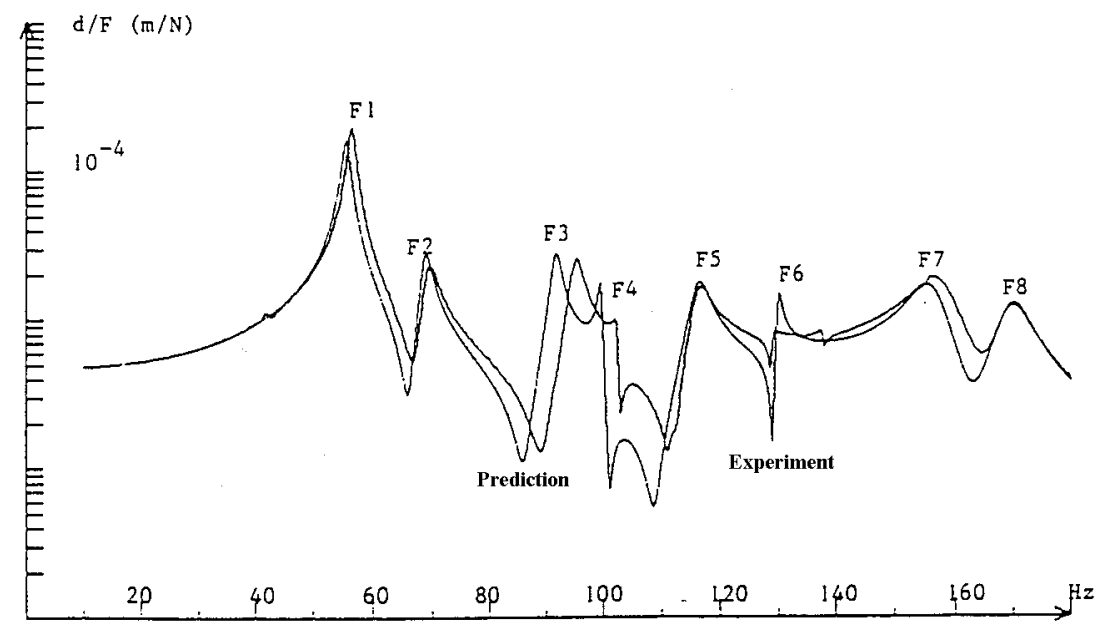

Fig. 6. Frequency response.

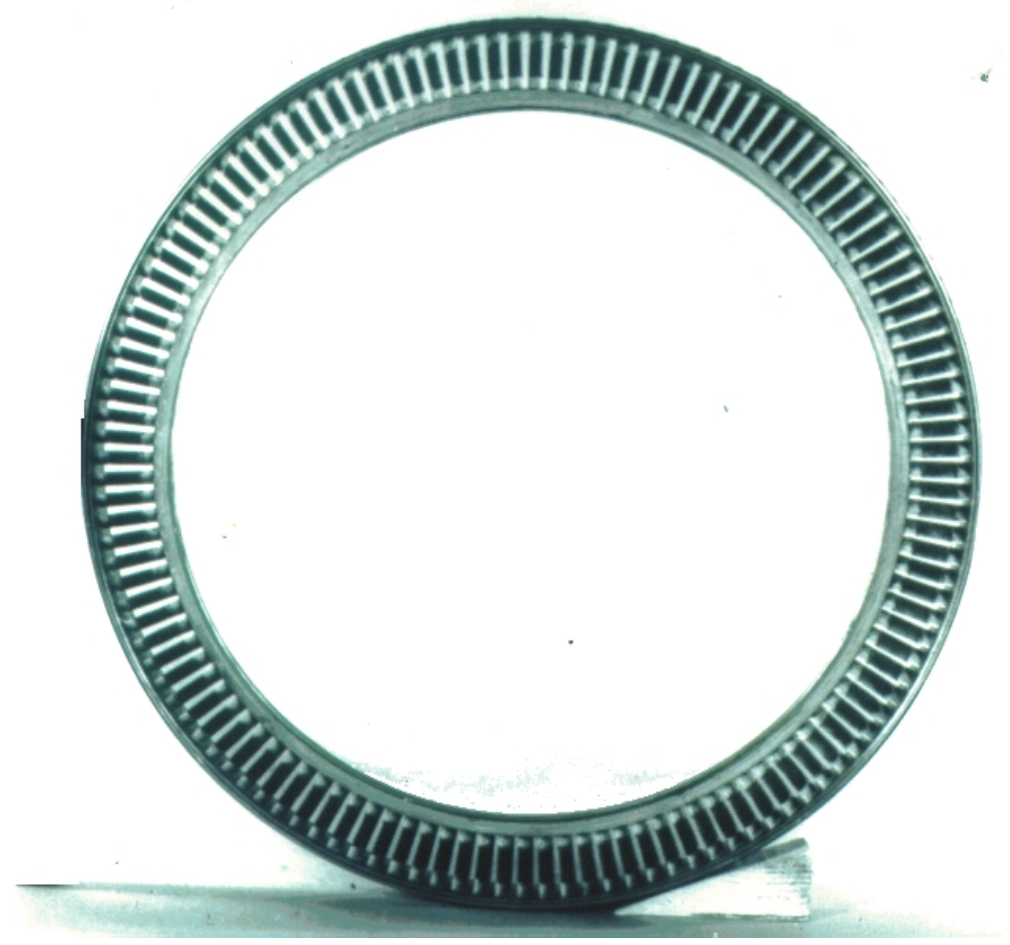

Fig. 7. Jet engine stator vane.

The application presented in [18] is a structure used for testing the method presented above, Fig. 5. Its finite element model uses more than 3000 degrees of freedom. The frequency response at a given point is presented in Fig. 6 which shows that the modal reduction (only 10 modes are used) is very efficient and that experimental and predicted results are in very close agreement.

A jet engine stator vane with blade ends coated with viscoelastic material for reducing the frequency response amplitude under resonant conditions is shown in Fig. 7. The prediction and experiments concerning mode 1 , loss factor and frequency, are shown in Fig. 8 [16]. 


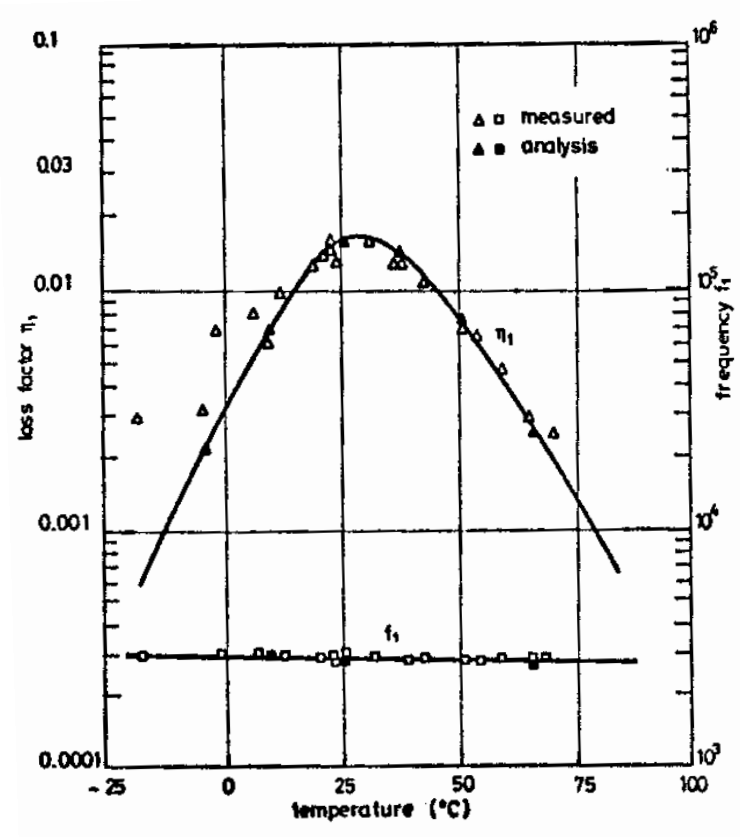

Fig. 8. loss factor and frequency.

Table 1

Frequencies and loss factors

\begin{tabular}{ccccc}
\hline Mode & \multicolumn{2}{c}{ Frequency $(\mathrm{Hz})$} & \multicolumn{2}{c}{ Loss factor } \\
& Experiment & Prediction & Experiment & Prediction \\
1 & 15.3 & 15.3 & 0.0085 & 0.0075 \\
2 & 59.2 & 59.4 & 0.0118 & 0.0102 \\
3 & 147.4 & 146.9 & 0.0102 & 0.0110 \\
4 & 274.7 & 279.3 & 0.0160 & 0.0164 \\
\hline
\end{tabular}

An alpine ski is presented in [17]. In normal use the center zone of the ski is pressed strongly into the snow and the stiffness of the shoe assembly is much greater than that of the ski. It is therefore of interest for ski manufacturers to determine the dynamic behavior of the front part of the ski. The results shown concern a ski made with 12 different metallic, composite and viscoelastic materials. The first four mode shapes are given in Fig. 9 and the frequencies and loss factors are given in Table 1.

\section{Examples of modeling damping due to simultaneous effects}

When damping results from several effects it is practically impossible to predict its value. However, when one deals with a family of structures, estimates on the quality factor $Q$ can be made. As for a one degree of
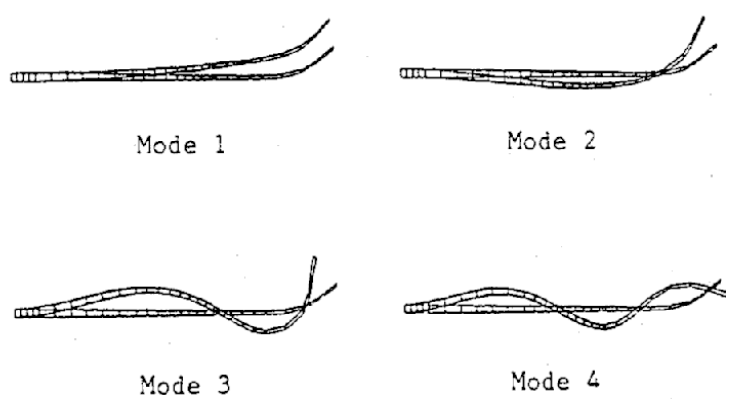

Fig. 9. Ski mode shapes.

freedom system, modal viscous damping coefficients are introduced by:

$$
c_{i}=\frac{1}{Q_{i}} \sqrt{k_{i} \cdot m_{i}}
$$

where $k_{i}, m_{i}$ and $Q_{i}$ are respectively the modal stiffness, the modal mass and the quality factor corresponding to the mode $i$. The situation can be such that the structure has already been built and can be tested in laboratory conditions.

A reciprocating single cylinder compressor was investigated [5]. The compressor unit is mounted on internal mounts, the stiffness of which is fairly known, and submitted to excitation forces due to the slidercrank mechanism. The compressor unit motion is defined by six degrees of freedom: 3 translations and 3 rotations of the center of inertia. The mass and stiffness matrices are known and modal mass and stiffness and modes shapes $m_{i}, k_{i}, \phi_{i}(i=1,6)$ of this undamped model are easily obtained. Then the $Q_{i}$ factors of the compressor unit, mostly depending on the oil bath and on the mounts, are obtained from an experimental frequency response. Equation (11) gives the modal damping ratios and $c_{i}, \ldots, c_{6}$ are the diagonal terms of the matrix

$$
c=\phi^{t} C \phi
$$

and as the matrix $\phi$ is square:

$$
C=\left(\phi^{t}\right)^{-1} c(\phi)^{-1}
$$

As the gyroscopic effect can be neglected equations (1) and (13) give:

$$
M \ddot{x}+\left(\phi^{t}\right)^{-1} c(\phi)^{-1} \dot{x}+K x=F(t)
$$

The comparison between experiments and prediction has shown good agreement, especially for the start-up and the steady-state motions.

Use of the quality factor obtained from experience and/or experiments is particularly important for aero- 


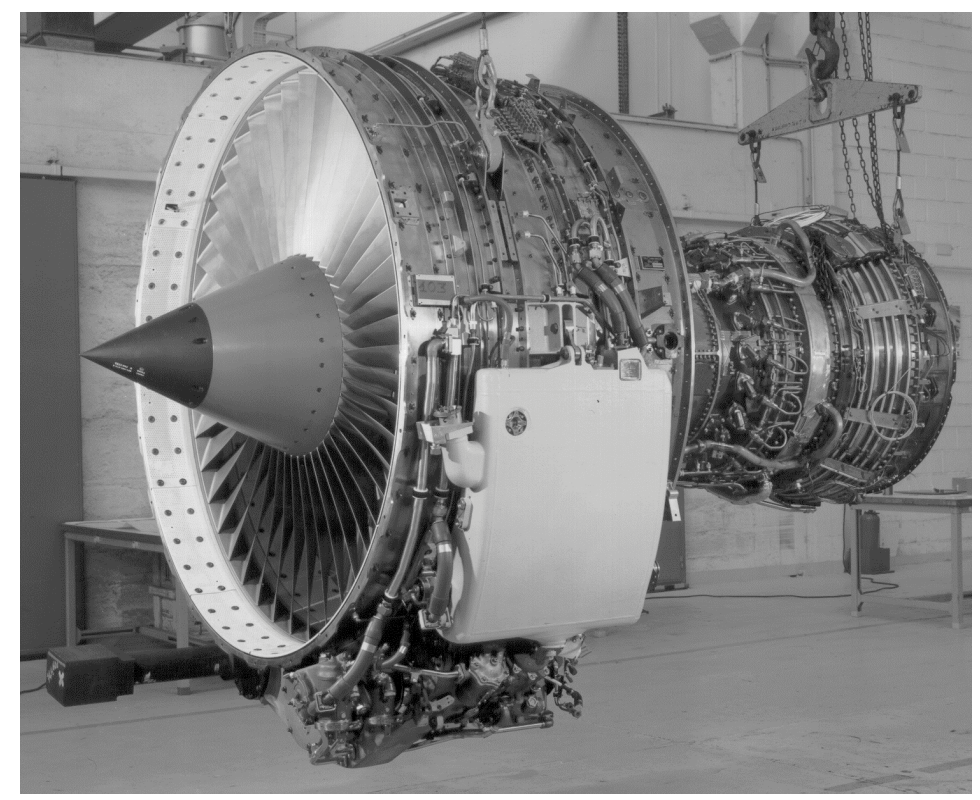

Fig. 10. CFM 56 jet engine - Courtesy SNECMA.

nautical structures which, for obvious reasons, must be very safe. Modeling must be very close to reality: the mass, stiffness and gyroscopic effects can be obtained quite easily, hence it is necessary to include the influ-

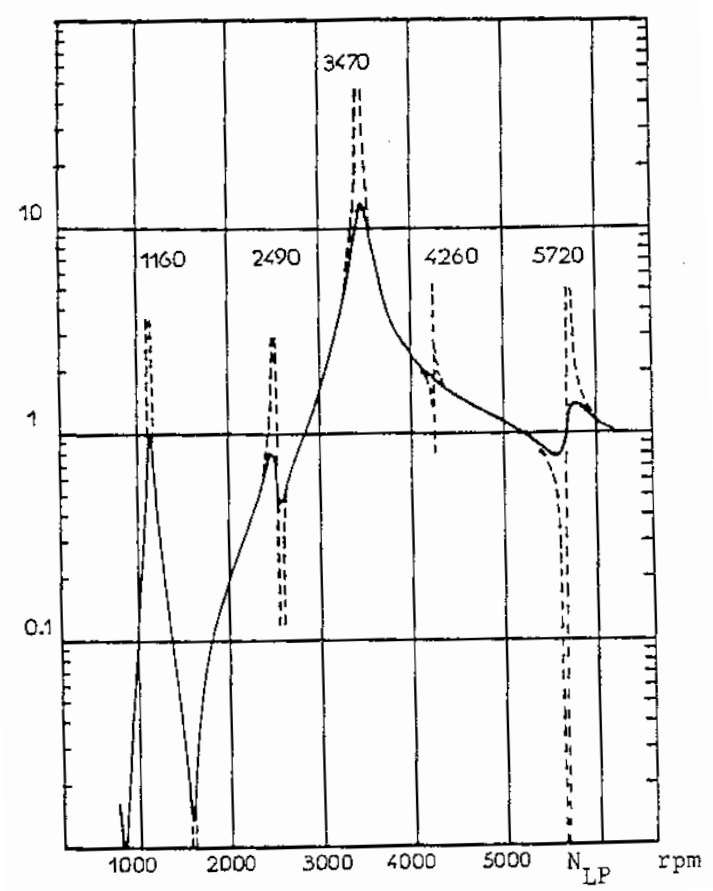

Fig. 11. Mass unbalance response. ence of damping in equations. Here damping comes from different physical mechanisms, such as friction, aerodynamics and material effects, acting simultaneously. These effects are difficult or practically impossible, at least at the present time, to model when dealing with engineering structures. Prediction of the dynamic behavior on the CFM-56 jet engine, see Figs 10 and 11 , was based of a model of the low pressure rotor, the high pressure rotor and the envelope [2]. The quality factor was given by the manufacturer who, at the time when the work was done, already had about ten years of experience on test rigs and results in flight conditions concerning dynamic behavior.

Results, concerning an air turbine starter [14], which are presented Figs 12 and 13 have been obtained with $Q$ factors coming from certain measurements made under operating conditions and from systematic measurements on the test rig shown in reference [4].

Reference [7] shows a propfan in a test rig which is used also for obtaining estimates on the quality factors.

\section{Conclusion}

If damping comes from only one effect it can often be included in equations; but experiments can be necessary. If damping comes from several effects an estimate can sometimes be made or, if the structure has been built, it can be measured. In any case when a new 


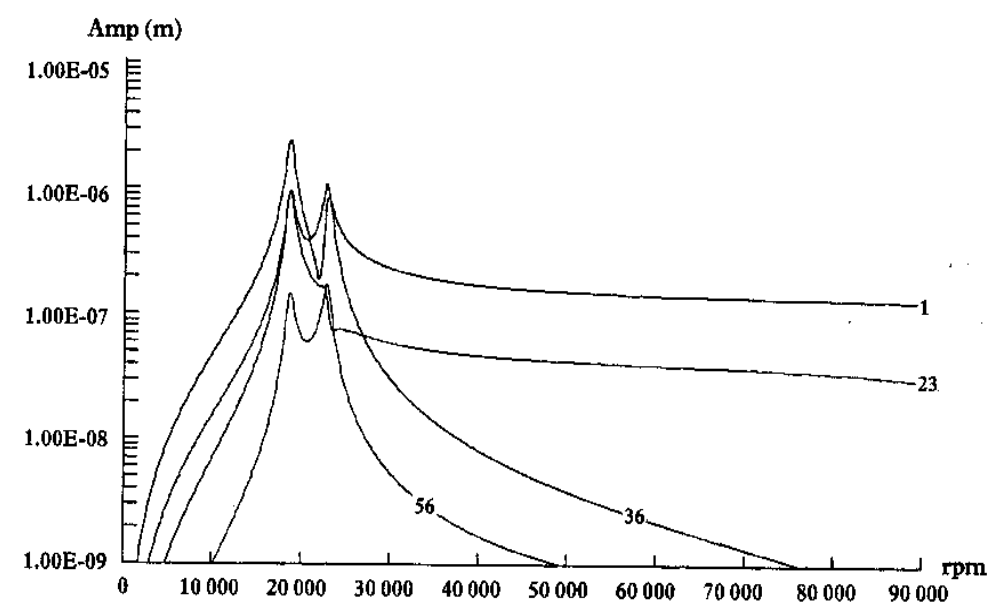

Fig. 12. Mass unbalance response (mass on rotor 1).

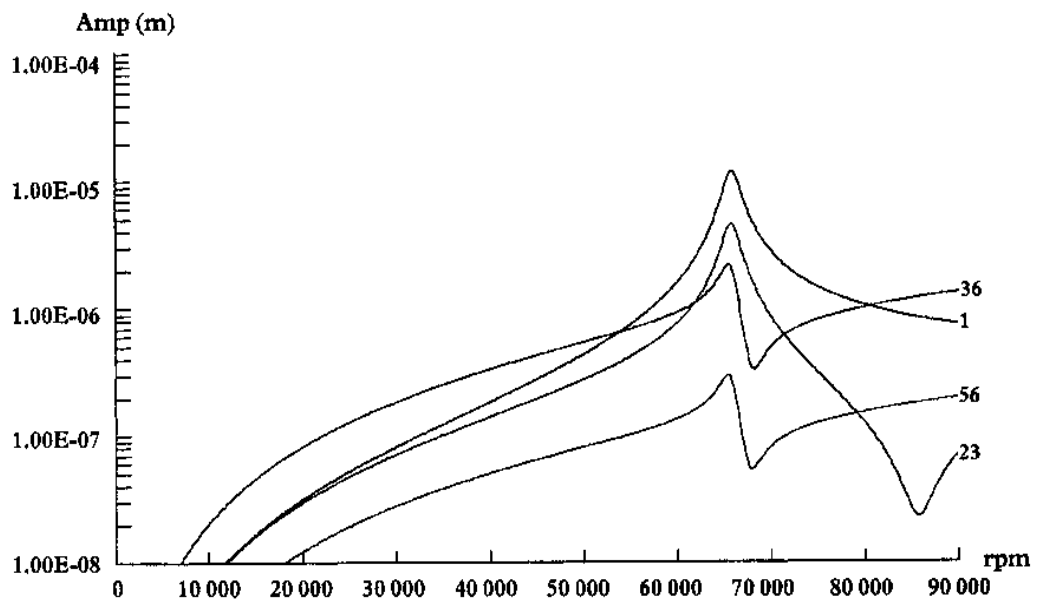

Fig. 13. Mass unbalance response (mass on rotor 2).

engineering structure with a damping effect difficult to model is concerned, it is important to carry out, simultaneously, modeling and experiments in situations close to operating conditions.

\section{References}

[1] A. Al Majid and R. Dufour, Un modèle forcé de restitution pour prévoir les réponses à des chocs d'une structure montée sur plot à friction, Mécanique Industrielle et Matériaux $\mathbf{5 1}$ (1998), 80-82.

[2] P. Berthier, G. Ferraris and M. Lalanne, Prédiction du comportement dynamique des moteurs d'avion: vitesses critiques, effets de balourd, J. de Mécanique Théorique et Appliquée 5 (1986), 573-585.

[3] D. Childs, Turbomachinery Rotordynamics, John Wiley, New York, 1993.
[4] A. Delbez, G. Charlot, G. Ferraris and M. Lalanne, Dynamic behavior of a counter-rotating air turbine stater, ASME Paper 93-GT-59, 1993.

[5] R. Dufour, J. Der Hagopian and M. Lalanne, Transient and steady state dynamic behavior of single cylinder compressors: prediction and experiments, J. of Sound and Vibration 181(1) (1995), 23-41.

[6] R. Dufour, M. Gérard and M. Charreyron, Dynamic Analysis of a crankshaft in bending with an electric motor and nonlinear fluid film bearings, IFToMM 5th International Conference on Rotordynamics, 1998, 200-211.

[7] G. Ferraris, V. Maisonneuve and M. Lalanne, Prediction of the dynamic behavior of non symmetric coaxial co-or counterrotating rotors, J. of Sound and Vibration 195(4) (1996), 649666.

[8] K. Gjika, R. Dufour and G. Ferraris, Transient response of structures on viscoelastic or elastoplastic mounts, prediction and experiment, J. of Sound and Vibration 198(3) (1996), 361-378. 
[9] K. Gjika and R. Dufour, Rigid body and non linear mount identification. Application to on board equipment with hysteretic suspension, J. of Vibration and Control 5 (1999), 75-94.

[10] M. Lalanne, M. Paulard and P. Trompette, Response of thick structures damped by viscoelastic material with application to layered beams and plate, The Shock and Vibration Bull $\mathbf{4 5}$ (1975), 65-72.

[11] M. Lalanne, P. Trompette, R. Henry and G. Ferraris, Analyse des Vibrations de moteurs. AGARD Conf. Proc. No 248, 1978.

[12] M. Lalanne, Progress in dynamic modeling. Science et défense 90, Dunod, Paris, 1990, pp. 360-367.

[13] M. Lalanne and G. Ferraris, Dynamique des rotors en flexion, Techniques de l'Ingénieur, Traité Génie Mécanique, B 5 110, 1996, pp. 1-36.
[14] M. Lalanne and G. Ferraris, Rotordynamics prediction in engineering, 2nd edition, John Wiley, Chichester, 1998.

[15] T, Someya, Journal bearing data book, Springer Verlag, Berlin, 1989.

[16] P. Trompette, M. Paulard, M. Lalanne, D.I.G. Jones and M.L. Parin, Prediction of modal damping of jet engine stator vanes using finite element techniques, ASME Paper 76-GT-60, 1976.

[17] C. Ulrich, G. Ferraris, M. Lalanne and J. Lacroix, Prediction of the frequency and time responses of composite structures: application to skis, ASME Book No G00825, 1993, pp. 9-13

[18] H.T. Zhou, J. Der Hagopian, G. Ferraris and M. Lalanne, Prediction of modal characteristics and harmonic response of viscoelastically damped structures, Shock and Vibration Symp. IV(59) (1988), 265-271. 

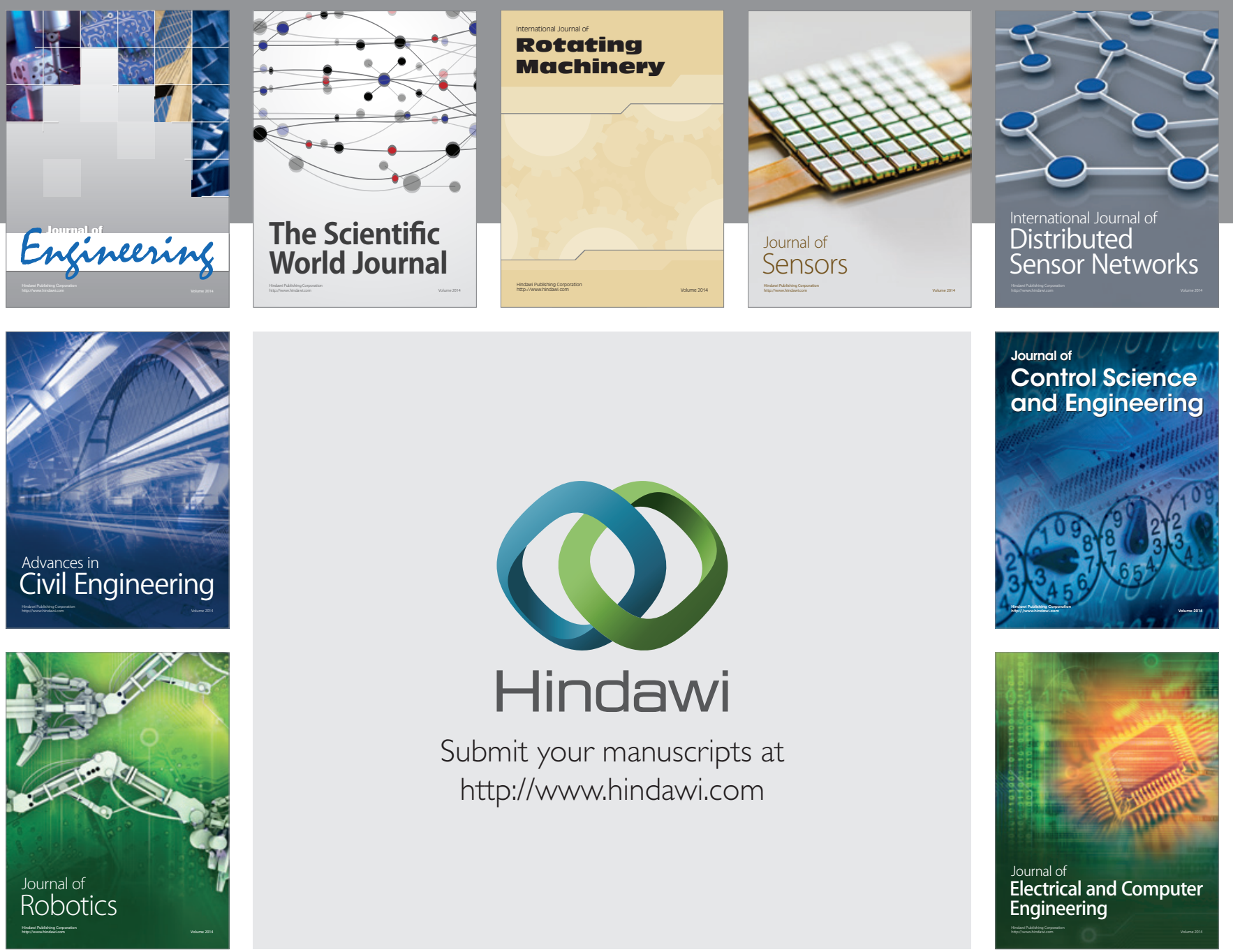

Submit your manuscripts at

http://www.hindawi.com
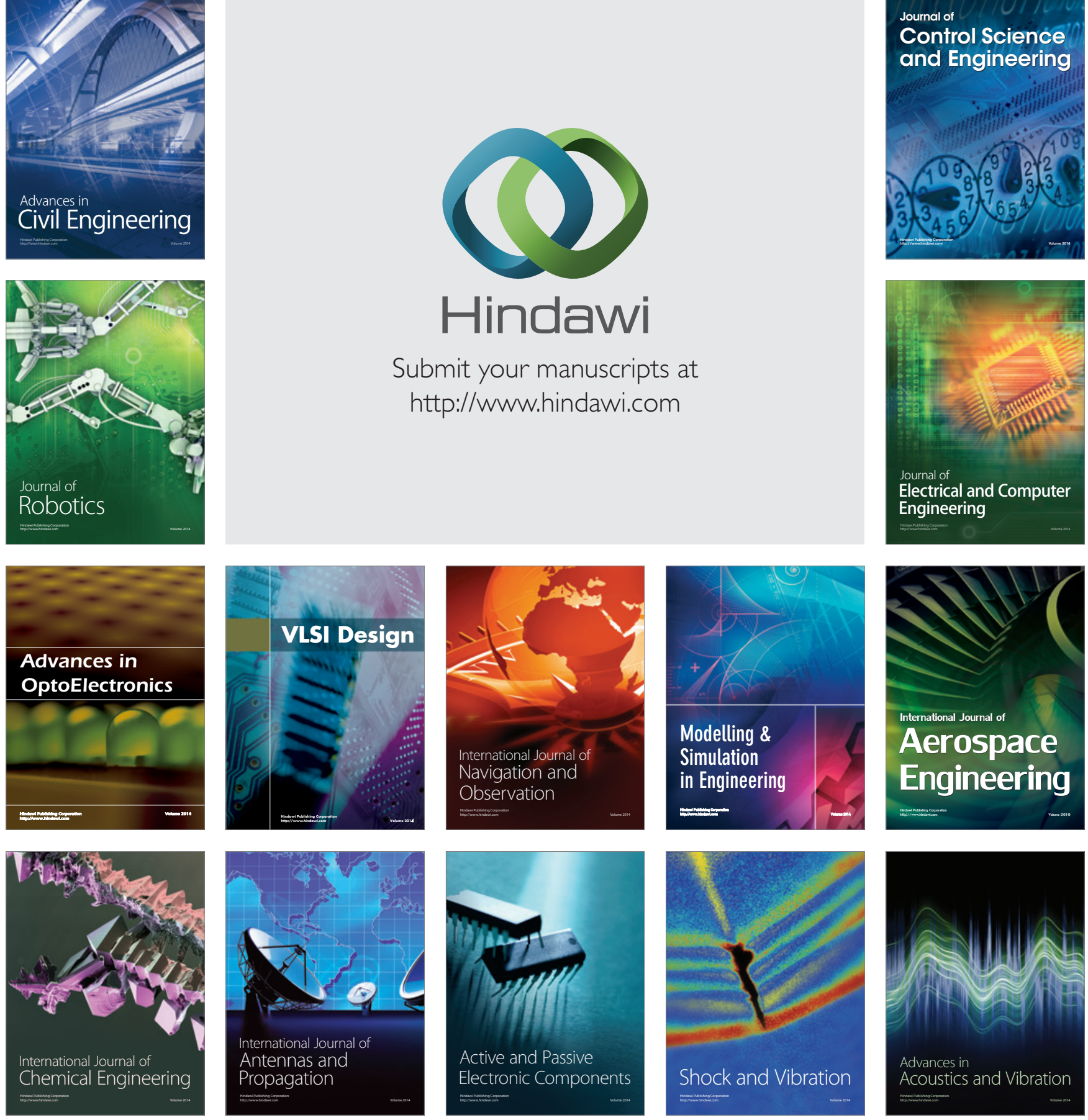